\title{
Prevalence of HPV infection among HIV-positive and HIV-negative women in Central/Eastern Italy: Strategies of prevention
}

\author{
EDOARDO TARTAGLIA ${ }^{1}$, KATIA FALASCA ${ }^{2}$, JACOPO VECCHIET ${ }^{2}$, GIOVANNA PAOLA SABUSCO $^{1}$, \\ GIOVANNA PICCIANO ${ }^{3}$, ROBERTO DI MARCO ${ }^{1}$ and CLAUDIO UCCIFERRI ${ }^{1}$ \\ ${ }^{1}$ Department of Medicine and Health Sciences 'Vincenzo Tiberio', University of Molise, I-86100 Campobasso; \\ ${ }^{2}$ Department of Medicine and Science of Aging-Clinic of Infectious Diseases, University 'G.' d'Annunzio Chieti-Pescara, \\ I-66100 Chieti; ${ }^{3}$ Department of Gynecology and Obstetrics, Cardarelli Hospital, I-86100 Campobasso, Italy
}

Received March 10, 2017; Accepted August 8, 2017

DOI: $10.3892 / \mathrm{ol} .2017 .7140$

\begin{abstract}
The present cross-sectional-study aimed to determine the prevalence of human papillomavirus (HPV)-genotypes among human immunodeficiency virus (HIV)-positive and -negative women in Central/Eastern Italy, and to identify the optimal strategies for effective HPV-prevention in each group. A representative sample of HIV-negative (150/200) and -positive (50/200) women, who underwent cervico-vaginal-swabbing. Swabs were analysed for a cytological screening and for a HPV-DNA-genotyping-test. A total of 66/200 swabs resulted HPV-positive. The overall HPV-prevalence was 33\% with a higher prevalence in the HIV-positive-group (48\%) compared with the HIV-negative-group (28\%). The most frequent genotypes were: $16,31,52,58,66,73$ and 89 . Furthermore, the prevalence of specific genotypes was different in each group. The results of the present study indicate that HIV infection appears to be an independent risk factor for HPV-infection. In addition, HPV-infection is more common and more likely to persist in HIV-positive compared with in HIV-negative women. The optimal way to counteract HPV infection is through primary prevention. The stage of immunity (cluster of differentiation 4-level) at the time of the HPV-screening is one of the most important parameters for detection of susceptibility to HPV-infection and to evaluate the response to the HPV-vaccine in HIV-positive women. It may be used to determine the sub-group of HIV-positive women that are more prone to HPV-infections or that exhibit a partial response
\end{abstract}

Correspondence to: Dr Edoardo Tartaglia, Department of Medicine and Health Sciences 'Vincenzo Tiberio', University of Molise, Viale Giovanni Paolo II (C.da Tappino), I-86100 Campobasso, Italy

E-mail: edo.tartaglia@libero.it

Key words: human papillomavirus, human immunodeficiency virus, risk factors, HPV-vaccine, HPV-prevalence to the HPV-vaccine. At present, a novel type of vaccine with 9-genotypes is available and in the near future, it may serve an essential role in the prevention of HPV infections.

\section{Introduction}

Human papillomavirus (HPV) is a DNA-virus, associated with epithelial hyperplasias. It is a small non-enveloped virus that contains a double-stranded, closed circular DNA genome, associated with histone-like proteins and protected by a capsid with icosahedron symmetry, formed by two structural protein types (1). It is included in the family of papillomaviridae, which actually contains 29 genera formed by 189 genotypes (2). Presently 170 genotypes, classified into five genera $(\alpha \mathrm{PV}, \beta \mathrm{PV}, \gamma \mathrm{PV}, \mu \mathrm{PV}, v \mathrm{PV})$, have been accepted to infect human being and approximately 40 of them, included in the genus Alphapapillomavirus, are associated with infections of the ano-genital and oral mucosa (3). One of the primary interests in HPV is to do with its unique oncogenic properties; so, genital HPV types usually are categorized according to their epidemiological association with cervical cancer in low- and high-risk (LR-HPV and HR-HPV).

Seven of them (HR-HPV: 16, 18, 31, 33, 45, 52, 58) account for nearly $90 \%$ of all cervical cancer cases in the general population worldwide with little regional variation and particularly genotypes 16 and 18 together account for the great majority of cervical carcinomas (70\%) in the general population (4). The HPV actually is estimated to be the most common sexually transmitted infection in the world (5) and its prevalence is seen in a geographic distribution of genotypes $(6,7)$.

Although HPV infection has a high transmission rate, more than $90 \%$ of new HPV infections, including those with high-risk types, clear or become undetectable within 24 months, and clearance usually occurs in the first 6 months after infection (8). Only $10 \%$ of women fail to clear HPV infections, resulting in a persistent infection. The main consequence of persistent infection with HR-HPV is the development of lesions that may progress to malignancy.

A high prevalence of new HPV infections and persistent infections are reported in immunocompromised individuals, 
including human immunodeficiency virus; human immunodeficiency virus (HIV)-positive women are at increased risk of incident HPV infection and reactivation of latent HPV infection, compared with HIV-negative women (9). Besides, HIV seropositivity has also been linked to high- and low-grade cervical squamous intraepithelial lesions (H-SIL and L-SIL), as well as invasive cervical carcinoma, a recognized AIDS-defining condition and a common cancer among HIV-positive women (10).

The aim of our study is to evaluate the prevalence of HPV infection in HIV infected females vs. HIV-negative women and its genotypes distribution in Central/Eastern Italy, using a HPV-DNA detection test, and to identify the best strategies for an effective HPV-prevention in both these groups of women.

\section{Materials and methods}

The authors have collected a representative sample of HIV-negative (150) and positive (50) women who attended the outpatients Departments of Gynecology and Obstetrics of University of Molise (Campobasso, Italy), of Infectious Diseases of University of Molise (Campobasso, Italy) and of University of Chieti-Pescara (Chieti, Italy), respectively, from January to July 2015 and who underwent cervico-vaginal swab collection. Swabs were analysed for a cytological screening (thinPrep System) and for HPV-DNA by polymerase-chain-reaction followed by type specific hybridization. HPV detection and typing was performed by using the Roche Linear Array HPV genotyping test (Roche Molecular Systems, Inc., Branchburg, NJ, USA) and it included probes for 37 HPV types.

Setting. Department of Medicine and Health Sciences, University of Molise and Department of Medicine and Science of Aging-Clinic of Infectious Diseases, University 'G. d'Annunzio' Chieti-Pescara.

Study population and design. This is a cross-sectional study. The study protocol received institutional review board (IRB), in accordance with the Code of Ethics of the Declaration of Helsinki. All females selected were eligible for participation to the study. Strobe statement and checklist have been consulted. Exclusion criteria was pregnancy.

Demographic and behavioral data. Nationality, race and ethnicity were reported. Grade of instruction, job/profession, marital status, smoke, drugs, use of alcohol were asked. Age at first sexual intercourse, lifetime number of sexual partners, sexual behaviour, use of oral-contraceptives, menopausal state and past history of pregnancy were reported. Height, weight and body mass index were evaluated.

Specimen collection and processing. Swabs were analyzed for a thin-Prep Pap test and for the presence of HPV-DNA by Linear Array HPV genotyping test, a qualitative in vitro test for the determination of 37 HPV-DNA genotypes. We considered as low-risk genotypes (LR-HPV): 6, 11, 40, 42, 54, 55, 61, 62, 64, 71, 72, 81, 83, 84, CP6108 (HPV-89) and as high-risk genotypes (HR-HPV): 16, 18, 26, 31, 33, 35, 39, 45, 51, 52, 53, 56, 58, 59, 66, 67, 68, 69, 70, 73, 82, IS39 (subtype of HPV-82).
Data on HIVinfection. Year of diagnosis, viral load(HIV-RNA), CD4 levels, CD4/CD8 ratio, CD4 nadir, eventual HCV/HBV co-infections, inflammatory indexes such as erythrocyte sedimentation rate (ESR) and C-reactive protein (CRP-test), CDC classification and eventual anti-retroviral therapy were collected. The cut-off point for $\mathrm{CD} 4^{+} \mathrm{T}$ lymphocytes in 200 cells $/ \mathrm{mm}^{3}$ was used to indicate immunosuppression in the women HIV positive.

Statistical analysis. All females who underwent an adequate swab for HPV evaluation were included in the final analysis (200/200). HPV prevalence was estimated within the $95 \%$ confidence interval (CI). CI were calculated by using the SE of $\log$ transformation with the SE of the log prevalence. Statistical analysis samples deemed positive for high- and low-risk HPV were categorized as single or multiple infections. To explore the association with age and overall HPV prevalence, age was categorized into 4 year intervals $(\leq 25,26-35,36-45, \geq 46)$. A post hoc power analysis was done using the $G^{*}$ Power statistical package for Windows (version 3.1.7; Microsoft Corporation, Redmond, WA, USA). The data were analyzed by an EpiInfo7 statistical package for Windows (version 7.1.0.6). For categorical variables, the Pearson's $\chi^{2}$ test and ANOVA test were performed to evaluate the significance of differences between groups. A $\mathrm{P}<0.05$ was considered statistically significant. A linear regression was performed to evaluate the relationship between variables.

\section{Results}

The study involved 200 women aged 18-66 years (mean, 36.45; SD, 11.12) by which 150 women (group 1) aged 18-63 years (mean, 34.34; SD, 10.99) were HIV-negative and 50 women (group 2) aged 23-66 years (mean, 42,80; SD, 8.94) were HIV-positive. The sample size of 200 was used for the statistical power analysis (in the ratio of 1 to 3 ). The alpha level used for this analysis was $\mathrm{P}<0.05$. The post hoc analyses revealed the statistical power for this study was $92 \%$ for detecting a medium effect. Thus, there was more than adequate power. The overall HPV prevalence was $33.00 \%$ (95\% CI: 27.16-39.41\%) with a higher prevalence in group 2, 48\% (95\% CI: 33.66-62.58\%) vs. group 1, $28 \%$ (95\% CI: 20.98-35.91\%) ( $\mathrm{P}=0.009)$ (Table I). Between the two groups there was a statistically significant difference of HPV prevalence among women across a board age range representative of the population $(\mathrm{P}=0.0027)$ and there was also a statistically significant difference of $\mathrm{HPV}$ prevalence related to $\mathrm{BMI}(\mathrm{P}=0.0089)$, marital status $(\mathrm{P}=0.0002)$ and education level $(\mathrm{P}=0.01)$ (Table I). There was also a statistically significant difference of HPV prevalence related to number of lifetime sex partners $(\geq 3)$, alcohol and drugs abuse with a prevalence of 54.12\% (95\% CI: 42.96-64.98\%) $(\mathrm{P}<0.0001)$, $72.73 \%$ (95\% CI: 39.03-93.98\%) (P=0.003) and 91.67\% (95\% CI: 61.52-99.79\%) $(\mathrm{P}<0.0001)$, respectively; but alcohol and drugs abuse was only observed in group 2 . There was also a statistically significant difference of HPV prevalence related to smoking $51.35 \%$ (95\% CI: 39.44-63.15\%) with a prevalence more statistically significant in group 2: $75.00 \%(95 \% \mathrm{CI}$ : $61.95-84.68 \%$ ) than in group 1: $40.00 \%$ (95\% CI: $28.41-52.82 \%)$ $(\mathrm{P}<0.0001)$. Particularly the prevalence of HR-HPV infection in smokers was $39.73 \%$ (95\% CI: 30.00-50.33\%) with a 
Table I. HPV prevalence and demographic data between groups.

\begin{tabular}{|c|c|c|c|c|c|c|c|}
\hline \multirow[b]{3}{*}{ Variable } & \multicolumn{3}{|c|}{ Group 1 (HIV negative) } & \multicolumn{3}{|c|}{ Group 2 (HIV positive) } & \multirow[b]{3}{*}{ P-value } \\
\hline & \multirow[b]{2}{*}{ Sample size } & \multicolumn{2}{|c|}{ HPV prevalence } & \multirow[b]{2}{*}{ Sample size } & \multicolumn{2}{|c|}{ HPV prevalence } & \\
\hline & & $\%$ & $(95 \% \mathrm{CI})$ & & $\%$ & $(95 \% \mathrm{CI})$ & \\
\hline HPV & 150 & 28.00 & $(20.98-35.91)$ & 50 & 48.00 & $(33.66-62.58)$ & 0.009 \\
\hline \multicolumn{8}{|l|}{ Age group } \\
\hline$\leq 25$ & 42 & 35.71 & $(23.55-50.04)$ & 2 & 50.00 & $(8.31-91.69)$ & 0.0027 \\
\hline $26-35$ & 39 & 30.77 & $(18.88-45.91)$ & 9 & 66.67 & $(41.58-84.89)$ & \\
\hline $36-45$ & 43 & 30.23 & (18.90-44.62) & 18 & 38.89 & $(21.00-60.37)$ & \\
\hline $46-55$ & 22 & 4.55 & $(0.64-26.11)$ & 18 & 50.00 & $(31.00-69.00)$ & \\
\hline$\geq 56$ & 4 & 25.00 & $(3.59-74.89)$ & 3 & 33.33 & $(4.94-82.78)$ & \\
\hline \multicolumn{8}{|l|}{ BMI group } \\
\hline$<18.5$ & 14 & 42.86 & $(22.37-66.12)$ & 1 & 0.00 & - & 0.0089 \\
\hline $18.5-24.9$ & 99 & 28.28 & $(20.59-37.50)$ & 30 & 53.33 & $(38.39-67.71)$ & \\
\hline $25-29.9$ & 29 & 20.69 & $(9.77-38.59)$ & 13 & 46.15 & $(24.57-69.28)$ & \\
\hline$>30$ & 8 & 25.00 & (6.61-61.09) & 6 & 33.33 & $(9.17-71.24)$ & \\
\hline \multicolumn{8}{|l|}{ Marital status } \\
\hline Single & 68 & 42.65 & $(32.50-53.46)$ & 33 & 63.64 & $(50.31-75.15)$ & 0.0002 \\
\hline Married/engaged & 82 & 15.85 & $(9.50-25.27)$ & 17 & 17.65 & $(5.91-42.24)$ & \\
\hline \multicolumn{8}{|l|}{ Education } \\
\hline Graduate & 20 & 25.00 & $(11.12-47.04)$ & 7 & 42.86 & $(16.24-74.36)$ & 0.01 \\
\hline High school & 82 & 34.15 & $(25.24-44.33)$ & 24 & 54.17 & (37.57-69.89) & \\
\hline Primary school & 48 & 18.75 & $(10.17-31.99)$ & 19 & 42.10 & $(24.15-62.42)$ & \\
\hline
\end{tabular}

HPV, human papillomavirus; HIV, human immunodeficiency virus.

higher prevalence in group 2 [62.50\% (95 CI: 46.65-76.06\%)] vs. group 1 [28.57\% (95\% CI: 18.09-42.01\%)] $(\mathrm{P}<0.0001)$. The frequency of vaccinated women for HPV was very low and it was observed only in group 1 (5/150): $3.33 \%$ (95\% CI: 1.39-7.75\%). No association was found between HPV infections and the age at the first intercourse, menopausal state, pregnancy, cancer familiarity, utilization of oral contraceptive. The odds ratio is shown in Fig. 1.

$H P V$-typing. Sixty-six out of two hundred swab resulted positive to HPV and twenty-five out of thirty-seven different HPV genotypes were detected (Fig. 2). Multiple HPV infections (29/66) were observed in $43.94 \%$ (95\% CI: $33.62-54.81 \%$ ) of HPV positive samples with a mean of 4 viruses for woman (range, 2-8). The most frequent genotypes detected were HPV-16, 31, 52, 58, 66, 73 and 89 (Fig. 2). Furthermore, the prevalence of specific genotypes was different in each group (Fig. 2) with a statistically significant higher prevalence of genotypes: $11,16,18$ and 31 in group 2 vs. group 1 with a prevalence of $10.00 \%$ (95\% CI: 3.33-21.81\%) $(\mathrm{P}=0.0007)$, $22.00 \%$ (95\% CI: $11.53-35.96 \%)(\mathrm{P}=0.0002), 8.00 \%(95 \%$ CI: $2.22-19.23 \%)(\mathrm{P}=0.016), 16.00 \%$ (95\% CI: $7.17-29.11 \%)$ $(\mathrm{P}=0.0001)$; respectively; An higher prevalence of genotypes: 52, 58 and 73 was detected in the group 1 vs. group 2 with a prevalence of $7.33 \%$ (95\% CI: 3.72-12.74\%) $(\mathrm{P}=0.17), 5.33 \%$ (95\% CI: $2.33-10.24 \%)(\mathrm{P}=0.70), 4.67 \%$ (95\% CI: $1.90-9.38 \%)$ $(\mathrm{P}=0.40)$; respectively; but it was not statistically significant.
The overall prevalence of HR-HPV genotypes was 24\% (95\% CI: $18.73-30.20 \%$ ) with a higher prevalence in HIV-Positive vs. HIV-negative women: $40 \%$ (95\% CI: 28.41-52.82\%) vs. $18.67 \%$ (95\% CI: $13.29-25.57 \%)$, respectively $(\mathrm{P}=0.002)$. The prevalence of HPV in relation to the presence of HIV infection (viral load, CD4 level, years from first diagnosis of AIDS and eventual anti-retroviral therapy) were reported in Table II.

\section{Discussion}

The Central/Eastern Italian regions are, mainly, a mountainous and agricultural land, so they are relatively isolated from the rest of country, and without presence of metropolitan areas; therefore there is a stability in HPV-genotype distribution (6). In these places, the burden of prevalence of HPV infection among females was overall greater than previous estimates in Italy: 33.0 vs. 7-16\% (official data of Italian Superior Health Institute) (11); moreover, our data, according with data of literature (12-14), showed an higher prevalence of HPV infection in HIV-seropositive (48.0\%) women compared to HIV-seronegative women (28.0\%).

HIV infection seems to be an independent risk factor for HPV-infection OR: 2.37 (95\% CI: 4.59-1.23\%). By contrast, the research into the role of genital HPV in HIV incident infection is currently limited, whereas it is biologically plausible (10). HPV could predispose to HIV infection and dissemination through its 


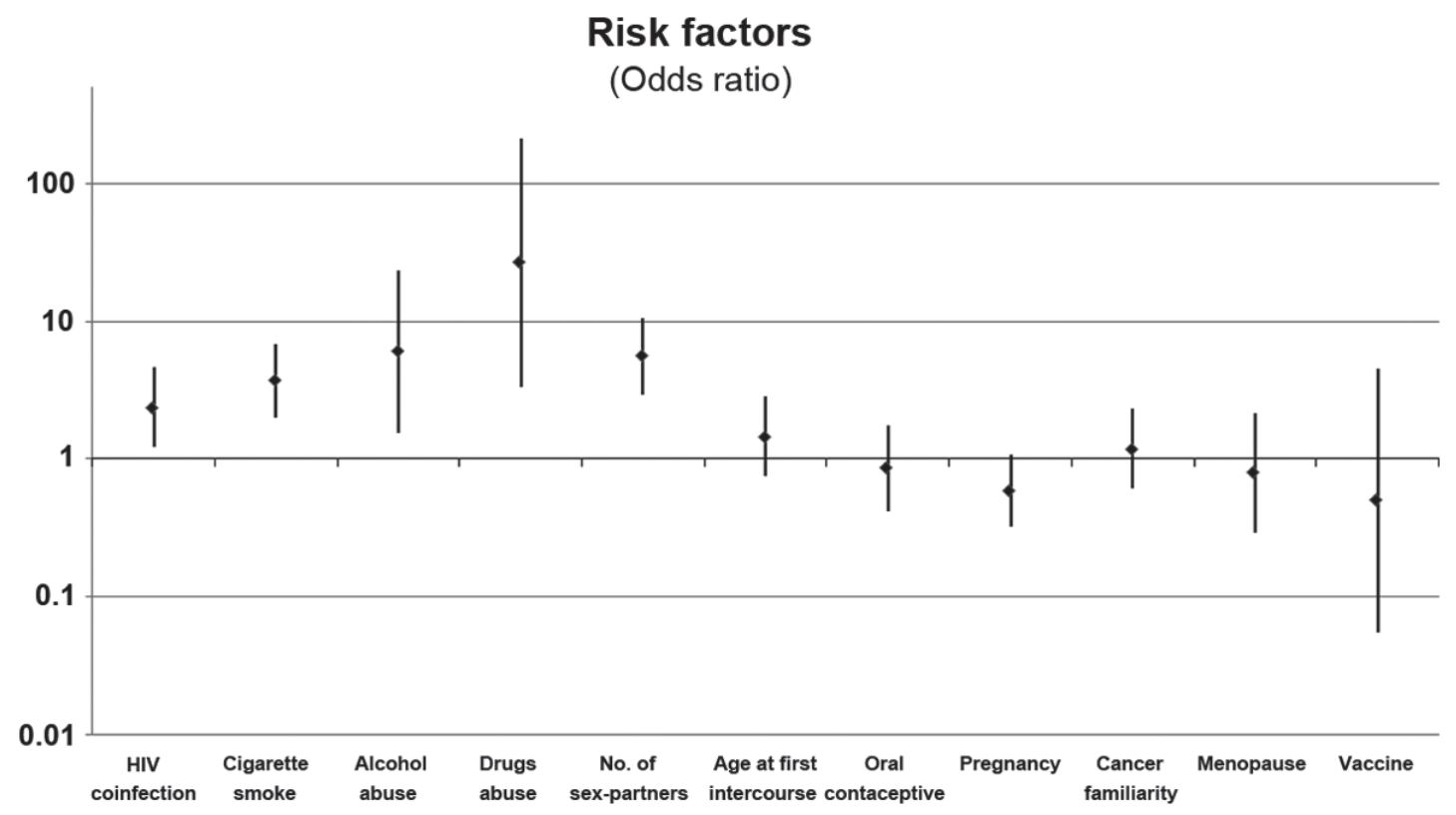

Figure 1. Risk factors (odds ratio). HIV, human immunodeficiency virus.

HPV prevalence

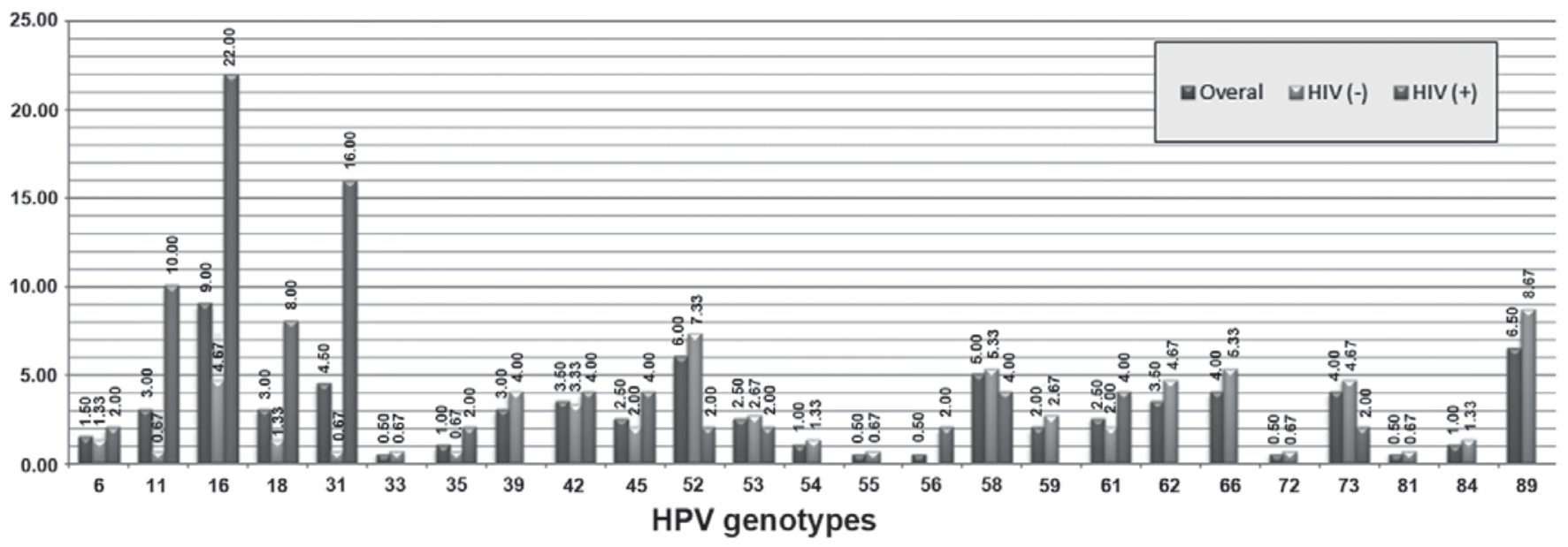

Figure 2. HPV prevalence (overall and into groups). HPV, human papillomavirus; HIV, human immunodeficiency virus.

disruption of the epithelium integrity and the mucosal immune system by altering the density and functional activities of epithelial Langerhans cells which capture infecting pathogens, enabling the recruitment and activation of HIV target cells such as T-lymphocytes, reducing the expression of proteins acting in cell adhesion and tumor suppression, downregulating proteins that promote the infiltration of Langerhans cells through the epithelium, reducing the production of proteins involved in antimicrobial activities and upregulating inflammatory cytokines, which may increase HIV replication (10). HIV infection is often associated with a greater drugs use (24\%), alcohol use (22\%) and smoking (48\%) which themselves may play a direct or indirect role in HPV infection; because alcohol and drugs abuse were only observed in group 2, it may be a bias and their role in HPV infection may be influenced by HIV co-infection. Whereas there was a strong statistically significant difference in HPV prevalence related to cigarette smoke $(\mathrm{P}<0.0001)$ and particularly, tobacco use was associated with HR-HPV infections. It is possible that smoking could increases the likelihood of HPV infection trough a local decrease of immune response in the cervix and an indirect effect related to metabolism of female hormones (15). The association with HIV infection may confer an increased susceptibility to the harmful effects of smoking (16).

Demographic and behavioural factors such as marital status, education level and number of sexual partners seem to influence the susceptibility to HPV infection, too. Particularly the results of multivariate analysis show that number of sexual partners in a lifetime and single status are independent risk factors for HPV infections. A statistically significant difference of HPV prevalence was also observed related to BMI $(\mathrm{P}=0.0089)$ and age groups $(\mathrm{P}=0.0027)$ with a lower prevalence of infection in the group of oldest and/or overweight women. No association was found between HPV infections and the 
Table II. HPV prevalence related to HIV infection.

\begin{tabular}{lccc}
\hline & & \multicolumn{2}{c}{ HPV prevalence } \\
\cline { 2 - 3 } Variable & Sample size & $\%$ & $(95 \%$ CI $)$ \\
\hline CD4 & & & \\
$<200$ & 7 & 57.14 & $(28.07-82.00)$ \\
$201-350$ & 4 & 50.00 & $(15.48-84.52)$ \\
$351-500$ & 15 & 73.33 & $(55.81-85.69)$ \\
$>500$ & 24 & 29.17 & $(15.07-48.87)$ \\
c-ART & & & \\
Yes & 42 & 47.62 & $(34.80-60.76)$ \\
No & 8 & 50.00 & $(23.14-76.86)$ \\
Viral load & & & $(35.87-76.07)$ \\
$<400$ & 14 & 57.14 & $(30.74-59.04)$ \\
$>400$ & 36 & 44.44 & \\
Years from diagnosis & & & $(15.39-64.22)$ \\
$<5$ & 11 & 36.36 & $(22.37-66.12)$ \\
$6-10$ & 14 & 22.37 & $(40.42-81.86)$ \\
$11-15$ & 11 & 40.42 & $(28.75-71.24)$ \\
$>15$ & 14 & 28.75 & 0.43 \\
\hline
\end{tabular}

HPV, human papillomavirus; HIV, human immunodeficiency virus.

age at the first intercourse, menopausal state, pregnancy, cancer familiarity, utilization of oral contraceptive. This last is in contrast with study of other authors (17) that found an association between oral contraceptive and HPV infection. But other previous researches (18) about the effect of oral contraceptive use on HPV infections show inconclusive results, too. Nonetheless, the findings in the present study do not support the hypothesis that oral contraceptive users may acquire HPV more often. The HPV-Vaccine seems to have a protective effect on HPV infection with OR: 0.5 (95\% CI: 0.0548-4.5646\%) but in this study, the frequency of vaccinated women for HPV was very low so at the moment it is impossible to evaluate correctly these data. In fact, the majority of whom were older than the population targeted for the vaccine. Our results show that a lower than $500 \mathrm{cell} / \mathrm{mm}^{3} \mathrm{CD} 4$ count was associated with higher HPV infections $(\mathrm{P}<0.05)$, thereby reflecting the inability of HIV-positive women's immune systems to respond to opportunist infection (14). Other studies suggested that the frequency of HPV persistence varied inversely with $\mathrm{CD}^{+}{ }^{+}$count and found higher HPV prevalence and incidence of oncogenic HPV types in HIV infected patients, especially those with lower CD4 ${ }^{+}$counts (19). These data suggest that the level of CD4 is important in the pathogenesis of HPV infection in HIV infected patients (19). No association was found with stage of HIV disease (CDC stage). In our study, we found that the stage of immunity (CD4-level) at the time of the HPV-screening is the most important parameter for detection of susceptibility to HPV-infection in HIV-positive women; nevertheless, it is very interesting to point up that the women with a low level of CD4 are those that only partially respond to HPV-vaccines (20). The other parameters that we tested in HIV patients such as viral load, ART and years from diagnosis is not related to HPV infection risk. Particularly, ART did not impact HPV detection within 6 months of therapy initiation, highlighting the importance of continued and consistent screening, even after ART-initiation and immune reconstitution (21).

In all these patients it is very important to perform preventively a primary prevention. The options for the primary prevention of HPV infections include HPV-vaccination and other interventions related to sexual health and behaviors, lifestyle habits (such as smoking) and others yet to be identified. These last interventions are most important, particularly in HIV-positive women, where the HPV-vaccination yet must be shown to be efficacious in HIV-infected individuals (20) particularly in the group with a CD4 count inferior to $500 \mathrm{cell} / \mathrm{mm}^{3}$. Secondly, another interesting aspect about vaccines issues from our study: the currently marketed vaccines do not provide total coverage against HPV infections; in fact, for the great variability of HPV circulating genotypes, not all most frequently circulating genotypes are included in 2-or 4-valent HPV vaccines (22-24) such as genotypes 31, more frequent in HIV-positive women, and $52 / 58$, more frequent in healthy women. This variability may be particularly dangerous in HIV-positive women where there is an increased risk of H-SIL and invasive cervical cancer related also to other HPV-genotypes different from HPV-16 and -18. But, at present, a new type of vaccine with 9 genotypes (HPV: 6, 11, 16, 18, 31, 33, 45, 52, 58) is available (9vHPV); it targets 5 additional cancer causing genotypes, which account for about $15 \%$ of cervical cancers and $25 \%$ of cervical pre-cancer lesions $(25,26)$, and preventing over more 95\% of persistent HPV31/33/45/52/58 infections and associated cervical, vulvar, and vaginal disease of any grade (27). The 9vHPV costs $\$ 13(12.30 €)$ more per dose than 4vHPV. 
The cost of the 9vHPV may be a limiting factor as well as the socio-political resistance to primary prevention (25-28). Yet, the 9-valent vaccination strategy would allow more aggressive changes in screening, and the incremental health benefits and medical costs averted from preventing cervical lesions and cervical cancer associated with HPV-types 31/33/45/52/58 further reducing future costs $(26,27)$. About the secondary prevention, the cervical-screening is still recommended in all women, included those vaccinated $(25,29)$.

In conclusion, HPV infection is the most common sexually transmitted infection in the world and its prevalence is seen in the geographic distribution of its genotypes (7). The most frequent genotypes detected in Central/Eastern Italy were HPV-16, HPV-89, HPV-52, HPV-58, HPV-31, HPV-66 and HPV-73. HPV infection is more common (particularly high-risk genotypes) and more likely to persist in HIV-positive women rather than HIV-negative women. The best way to counteract the HPV infection is the primary prevention that includes HPV-vaccination and other interventions related to lifestyle habits, sexual health and behaviors. These last behavioural interventions are most important, particularly in HIV positive women, where the HPV-vaccination yet must be shown to be efficacious in HIV-infected individuals (20), particularly in the group with a low CD4 count. In fact, the women with a low level of CD4 are those that only partially respond to HPV-vaccines (20); furthermore, in our study, we confirmed that the stage of immunity (CD4 level) at the time of the HPV screening is the most important parameter for detection of susceptibility to HPV-infection in HIV-positive women. So, it may be used to check the sub-group of HIV positive women that are more exposed to HPV infections and to its complications and that have a partial response to HPV-vaccine. However, for the great variability of HPV circulating genotypes, currently, the secondary prevention is still recommended in all women, included those vaccinated, and particularly in HIV-positive women $(25,29)$. Nevertheless, at present, a new type of vaccine with 9 genotypes is available; we believe that, in the near future, it will have a key role to play in the prevention of HPV infections, indeed it may be useful for assuring a larger vaccine coverage across the population, reducing the circulating genotypes in the overall population and, indirectly, it may also be useful in protecting those patients that presently respond partially or do not respond at all to vaccine. So, if larger coverage represents an opportunity for each woman, so much more so for every HIV-positive woman; it is mandatory.

\section{Acknowledgements}

The authors would like to thank Simon Rees for his help revising this text before publication.

\section{References}

1. Lazarczyk M, Cassonnet P, Pons C, Jacob Y and Favre M: The EVER proteins as a natural barrier against papillomaviruses: A new insight into the pathogenesis of human papillomavirus infections. Microbiol Mol Biol Rev 73: 348-370, 2009.

2. Bernard HU, Burk RD, Chen Z, van Doorslaer K, zur Hausen $H$ and de Villiers EM: Classification of papillomaviruses (PVs) based on 189 PV types and proposal of taxonomic amendments. Virology 401: 70-79, 2010.
3. de Villiers EM: Cross-roads in the classification of papillomaviruses. Virology 445: 2-10, 2013.

4. McKenzie ND, Kobetz EN, Ganjei-Azar P, Rosa-Cunha I, Potter JE, Morishita A, Lucci JA III, Guettouche T, Hnatyszyn JH and Koru-Sengul T: HPV in HIV-infected women: Implications for primary prevention. Front Oncol 4: 179, 2014.

5. Dunne EF, Unger ER, Sternberg M, McQuillan G, Swan DC, Patel SS and Markowitz LE: Prevalence of HPV infection among females in the United States. JAMA 297: 813-819, 2007.

6. Tartaglia E, Iafusco D, Galderisi A and Mastrantonio P: Do HPV vaccine genotypes agree with HPV circulating types? Lancet Infect Dis 11: 585-586, 2011.

7. Tartaglia E, Iafusco D, Cocca A, Palomba S, Rotondi M and Mastrantonio P: HPV at the time of Vaccine: Has screening reached its goal? Eur J Gynecol Oncol 33: 591-597, 2012.

8. Franco EL, Villa LL, Sobrinho JP, Prado JM, Rousseau MC, Désy $\mathrm{M}$ and Rohan TE: Epidemiology of acquisition and clearance of cervical human papillomavirus infection in women from a high-risk area for cervical cancer. J Infect Dis 180: 1415-1423, 1999.

9. Mbulawa ZZ, Marais DJ, Johnson LF, Coetzee D and Williamson AL: Impact of human immunodeficiency virus on the natural history of human papillomavirus genital infection in South African men and women. J Infect Dis 206: 15-27, 2012.

10. Lissouba P, Van de Perre P and Auvert B: Association of genital human papillomavirus infection with HIV acquisition: A systematic review and meta-analysis. Sex Transm Infect 89: 350-356, 2013.

11. Filia A: Epidemiological aspects of HPV infection (last revision Apr 1, 2015). http://www.epicentro.iss.it/problemi/hpv/epid.asp. Accessed Feb 23, 2015.

12. Madeddu G, Mameli G, Capobianco G, Babudieri S, Maida I, Bagella P, Rocca G, Cherchi PL, Sechi LA, Zanetti S, et al: HPV infection in HIV-positive females: The need for cervical cancer screening including HPV-DNA detection despite successful HAART. Eur Rev Med Pharmacol Sci 18: 1277-1285, 2014.

13. Clifford GM, Goncalves MA and Franceschi S; HPV and HIV Study Group: Human papillomavirus types among women infected with HIV: A meta-analysis. AIDS 20: 2337-2344, 2006.

14. Camargo M, Soto-De Leon SC, Munoz M, Sanchez R, PeñaHerrera D, Pineda-Peña AC, Sussmann O, Paez C,Perez-Prados A, Patarroyo ME and Patarroyo MA: Human papillomavirus detection in women with and without human immunodeficiency virus infection in Colombia. BMC Cancer 14: 451, 2014.

15. Muñoz N, Castellsagué X, de González AB and Gissmann L: HPV in the etiology of human cancer. Vaccine 24 (Suppl 3) S3/1-10, 2006.

16. Calvo M, Laguno M, Martínez M, Martínez E: Effects of tobacco smoking on HIV-infected individuals. AIDS Rev 17: 47-55, 2015.

17. Cotton SC, Sharp L, Seth R, Masson LF, Little J, Cruickshank ME, Neal K and Waugh N; TOMBOLA Group: Lifestyle and socio-demographic factors associated with high-risk HPV infection in UK women. Br J Cancer 97: 133-139, 2007.

18. Green J, Berrington de Gonzalez A, Smith JS, Franceschi S, Appleby P, Plummer M and Beral V: Human papillomavirus infection and use of oral contraceptives. Br J Cancer 88: 1713-1720, 2003.

19. Silva Ld, Miranda A, Batalha R, Ferreira L, Santos M and Talhari S: High-risk human papillomavirus and cervical lesions among women living with HIV/AIDS in Brazilian Amazon, Brazil. Braz J Infect Dis 19: 557-562, 2015.

20. Denny LA, Franceschi S, de Sanjosé S, Heard I, Moscicki AB and Palefsky J: Human papillomavirus, human immunodeficiency virus and immunosuppression. Vaccine 30 (Suppl 5): F168-F174, 2012.

21. Rositch AF, Gravitt PE, Tobian AA, Newell K, Quinn TC, Serwadda D, Ssebbowa P, Kiggundu V, Gray RH and Reynolds SJ: Frequent detection of HPV before and after initiation of antiretroviral therapy among HIV/HSV-2 co-infected women in Uganda. PLoS One 8: e55383, 2013.

22. Hong H, He TF, Ni HX, Zhang S and Xu GZ: Prevalence and genotype distribution of HPV infection among women in Ningbo, China. Int J Gynaecol Obstet 131: 96-99, 2015.

23. Sammarco ML, Ucciferri C, Tamburro M, Falasca K, Ripabelli G and Vecchiet J: High prevalence of human papillomavirus type 58 in HIV infected men who have sex with men: A preliminary report in Central Italy. J Med Virol 88: 911-914, 2016.

24. Liu G, Markowitz LE, Hariri S, Panicker G and Unger ER: Seroprevalence of 9 human papillomavirus types in the United States, 2005-2006. J Infect Dis 213: 191-198, 2016. 
25. Petrosky E, Bocchini JA Jr, Hariri S, Chesson H, Curtis CR, Saraiya M, Unger ER and Markowitz LE; Centers for Disease Control and Prevention (CDC): Use of 9-valent human papillomavirus (HPV) vaccine: Updated HPV vaccination recommendations of the advisory committee on immunization practices. MMWR Morb Mortal Wkly Rep 64: 300-304, 2015.

26. Chesson HW, Laprise JF, Brisson M and Markowitz LE: Impact and cost-effectiveness of 3 doses of 9-Valent human papillomavirus (HPV) vaccine among US females previously vaccinated with 4-valent HPV vaccine. J Infect Dis 213: 1694-1700, 2016.
27. Brisson M, Laprise JF, Chesson HW, Drolet M, Malagón T, Boily MC and Markowitz LE: Health and economic impact of switching from a 4-valent to a 9-valent HPV vaccination program in the United States. J Natl Cancer Inst 108: pii: djv282, 2015.

28. Kojic EM and Rana AI and $\mathrm{Cu}-U y i n$ S: Human papillomavirus vaccination in HIV-infected women: Need for increased coverage. Expert Rev Vaccines 15: 105-117, 2016.

29. Zhai L and Tumban E: Gardasil-9: A global survey of projected efficacy. Antiviral Res 130: 101-109, 2016. 